\title{
Exploring the Relationship Between Endometriosis and Vascular Health: A Systematic Mini-Review
}

\author{
Santos JM ${ }^{1}$, Singh $\mathrm{B}^{2}$ and Xia $\mathrm{P}^{3 *}$ \\ ${ }^{1}$ Department of Post-Baccalaureate Premedical Program, USA \\ ${ }^{2} J o h n s$ Hopkins Medicine, Research \\ ${ }^{3}$ Department of Gynecology and Obstetrics, Baltimore, USA
}

*Corresponding author: Ping Xia, Department of Gynecology and Obstetrics, Johns Hopkins Medicine, Baltimore, Maryland, USA

ARTICLE INFO

Received: 蔧 March 07, 2019

Published: March 18, 2019

Citation: Santos JM, Singh B, Xia P. Exploring the Relationship Between Endometriosis and Vascular Health: A Systematic Mini-Review. Biomed J Sci \& Tech Res 16(1)-2019. BJSTR. MS.ID.002798.

\begin{abstract}
The relationship between endometriosis and vascular health has been postulated over the past couple of years. Because the pathophysiology of endometriosis remains somewhat unclear, there is great interest in this relationship. Due to the co-current nature of endometriosis, vasculature, and cardiovascular disease, several therapies geared toward vascular-related processes (i.e. angiogenesis and atherosclerosis) have been proposed and/or studied. In this review paper, we discuss the evidence of five articles that support the correlation between endometriosis and vascular health and its clinical implications.
\end{abstract}

\section{Introduction}

Endometriosis affects up to $50 \%$ of women with infertility [1]. It is a costly disease with an estimated annual economic burden in the United States of $\$ 78.05$ billion in 2013 [2]. It is the presence of endometriotic lesions in extrauterine locations (e.g. pelvic peritoneum, ovaries, rectovaginal septum [3]. For the endometriotic lesions to plant itself and survive, it must establish its own blood supply- thus vascularization (i.e. angiogenesis) is a major hallmark in the pathogenesis of endometriosis [4]. Angiogenesis is initiated by angiogenic growth factors (e.g. vascular endothelial growth factor, VEGF), which have been extensively studied as it is heavily expressed in red endometriotic lesions [4]. In addition to angiogenesis, cardiovascular disease (e.g. atherosclerosis) has shown potential in characterizing endometriosis since it is a source of systemic subclinical inflammation [5]. Therefore, because of the developing correlation between endometriosis and vascular, interest in developing treatments focused on anti-angiogenics and anti-inflammatories have grown.

\section{Materials and Methods}

\section{Search and Study Selection}

In order to find clinical trials and/or review papers that support the relationship between endometriosis and vascular health and possible treatments, the decision was made to search PubMed. Because of the focus on the relationship between these two factors, the search term "endometriosis and vascular health" was used. This initially garnered 336 results, which were further filtered by publication dates within ten years. This filter narrowed down the search to 131 articles, which varied between clinical trials and review papers.

\section{Eligibility Criteria}

In order to be eligible for review, the clinical trial and/or review paper had to be published within the past ten years to ensure relevancy of data and information that could be translated for current use. Furthermore, the title of the articles had to include 
endometriosis along with terms related to vascular health (e.g. antio-angiogenic, statin, vascular, atherosclerosis).

\section{Results}

Amongst the systemic search from PubMed, five articles were eligible and included in this review paper. Two discussed the relationship between endometriosis and vascular health, while the remaining three discussed treatment options that focused on a vascular cause (i.e. anti-angiogenics and anti-inflammatories). Of the five, four were review papers. The remaining article was a study. Two of the review papers discussed vascularization and atherosclerosis in the context of endometriosis, while the other two review papers discussed treatment options that focused on vascularization. The study focused on proposing and supporting a vascular-associated treatment.

\section{Discussion}

\section{Angiogenesis}

MicroRNAs (miRNAs) may play important role in signaling network regulating angiogenesis in endometriosis. Some have reported that they can control expression of angiogenic factors [6]. Furthermore, vasculogenesis incorporates circulating endothelial progenitor cells (EPCs) from the bone marrow into the microvascular endothelium of newly developing micro vessels. These EPCs contribute to the formation of new blood vessels (e.g. tumors, MIs, stroke) as well as vascularization of endometrium. Often times EPCs are recruited in the microvasculature of endometriotic lesions and regulated by VEGF and possible estrogen. Therefore, a potential treatment would focus on gene therapy (e.g. miRNAs) targeting angiogenesis and reducing the micro vessel density of endometriotic lesions. Traditional pharmacological and surgical therapies (e.g. oral contraceptives, excision of endometriotic lesions) can be effective, but only temporarily. Recurrence is often high after cessation of contraceptives (and similar estrogen suppressing medications) and even after surgery. Endometriotic lesions thrive when there is angiogenesis (like tumors and Mets) due to their dependency on a enough blood supply.

Therefore, anti-angiogenic compounds could potentially be a viable treatment option. A current therapeutic approach to preventing angiogenesis, which is crucial in the development of endometriosis, is to pair an angiogenic cell (using a cell specific surface molecule) with its killing agent. Hu analyzed tissue factor VII (VII-TF) as the cell specific surface molecule on angiogenic vascular endothelial cells (VEC). As discussed in Laschke \& Menger, anti-angiogenic therapies are at a somewhat standstill due to the lack of specificity (i.e. anti-angiogenic target molecule). However, identification of factor VII-TF on VECs has led to promising therapies such as, immunotherapy and photodynamic therapy [6].

\section{Cardiovascular Health (e.g. Atherosclerosis)}

There is a possible correlation between cardiovascular disease (e.g. atherosclerosis) and endometriosis. Although the pathophysiology of endometriosis remains somewhat unclear, oxidative stress, and subsequent inflammation, may play a role. Systemic subclinical inflammation, possibly originating from atherosclerosis, has been known to help characterize endometriosis. As a result, studies have found that statins, due to their inhibitive and disruptive properties of certain enzymes and enzymatic pathways, have potential to inhibit the growth of endometrial stromal cells. Because collagen contributes to the formation of scar tissue (fibrosis) associated with endometriosis, statins reduced the contraction of and binding of endometrial stromal cells to collagen fibers. Furthermore, simvastatin was found to induce apoptosis of human endometrial stromal cells. High doses of statins, specifically atorvastatin, were found to reduce endometrial implants and subsequently inhibit angiogenesis.

\section{Conclusion}

Although anti-angiogenics are a promising candidate for endometriosis treatment, it comes with complications. Since most patients affected by endometriosis are women of childbearing age, anti-angiogenic compounds need to be specialized towards inhibiting angiogenesis in endometriotic lesions and not in female structures such as, the ovary, placenta and uterus. Accordingly, anti-angiogenic treatments can be teratogenic and impair fertility. Furthermore, even if the anti-angiogenic treatment can inhibit a specific angiogenic growth factor, it may not be able to inhibit another compensating angiogenic growth factor. Therefore, these types of treatments may be effective towards the beginning stages of endometriosis or after surgical intervention by reducing recurrence.

In addition to angiogenesis, another apparent comorbidity of endometriosis is cardiovascular disease, specifically atherosclerosis. As discussed in Santoro atherosclerosis causes systemic subclinical inflammation that characterizes endometriosis.

Gibram found that statins, which are anti-inflammatories, have the potential to not only treat atherosclerosis, but also inhibit the growth of endometrial stromal cells and angiogenesis. Therefore, statins could be a possible treatment for endometriosis since they have anti- inflammatory and antioxidant properties. In conclusion, although there were limited clinical trials and review papers obtained in the systematic search, there is promising potential of a relationship between endometriosis and vascular health. As a result, new therapies for endometriosis are starting to be geared toward treating vascularization (i.e. anti-angiogenics and anti-inflammatories). More clinical trials and studies need to be performed in order to strengthen this relationship and its clinical implications.

\section{References}

1. Gibran L, Maranhao R, Abrao M, Baracat E, Podgaec S (2014) Could statins constitute a novel treatment for endometriosis? Systematic review of the literature. European Journal of Obstetrics \& Gynecology and Reproductive Biology 179: 153-158. 
2. Hu Z, Cheng J, Xu J, Ruf W, Lockwood C (2017) Tissue factor is an angiogenic-specific receptor for factor VII-targeted immunotherapy and photodynamic therapy. Angiogenesis 20(1): 85-96.

3. Laschke MW, Menger MD (2018) Basic mechanisms of vascularization in endometriosis and their clinical implications. Human Reproduction Update 24: 207-224.

4. Laschke M, Menger M (2012) Anti-angiogenic treatment strategies for the therapy of endometriosis. Human Reproduction Update 18(6): 682 702 .

\section{ISSN: 2574-1241}

DOI: 10.26717/BJSTR.2019.16.002798

Xia P. Biomed J Sci \& Tech Res

(C) This work is licensed under Creative

Submission Link: https://biomedres.us/submit-manuscript.php
5. Santoro L, D Onofrio F, Flore R, Gasbarrini A, Santoliquido A (2015) Endometriosis and atherosclerosis: what we already know and what we have yet to discover. American Journal of Obstetrics and Gynecology 213(3): 326-331.

6. Soliman A, Yang H, Du E, Kelley C, Winkel C (2016) The direct and indirect costs associated with endometriosis: a systemic literature review. Human Reproduction 31(4): 712-722.

$\begin{array}{ll}\text { BIOMEDICAL } & \text { Assets of Publishing with us } \\ \text { RESEARCHES } & \text { - Global archiving of articles } \\ \text { ISSN: } 2574-1241 & \text { - Immediate, unrestricted online access } \\ \end{array}$

